\title{
A Photonic Nanojet as Tunable and Polarization-Sensitive Optical Tweezers
}

\author{
Aleksandr Kovrov, Andrey Novitsky, Alina Karabchevsky, and Alexander S. Shalin*
}

The ability to manipulate small objects with focused laser beams has opened a venue for investigating dynamical phenomena relevant to both fundamental and applied sciences. However, manipulating nano-sized objects requires subwavelength field localization, provided by auxiliary nano- and microstructures. Particularly, dielectric microparticles can be used to confine light to an intense beam with a subwavelength waist, called a photonic nanojet (PNJ), which can provide sufficient field gradients for trapping nano-objects. Herein, the scheme for wavelength-tunable and nanoscale-precise optical trapping is elaborated, and the possibility of lateral nanoparticle movement using the PNJ's side lobes is shown for the first time. In addition, the possibility of subwavelength positioning using polarization switching is shown. The estimated stability with respect to Brownian motion is higher compared to conventional optical trapping schemes.
One promising and already proven concept for improving optical tweezers is to employ auxiliary plasmonic and dielectric nanostructures, which can generate strong near-fields. ${ }^{[1-11]}$

Specifically, additional degrees of freedom for optical manipulation could be provided by dielectric microparticles and their ensembles, generating photonic nanojets (PNJs), ${ }^{[12]}$ whose shape depends on the microparticle's shape. ${ }^{[13-19]}$

PNJs can trap any nanoparticle, including metallic ones, in spite of repulsive scattering forces (radiation pressure, which is much stronger for metallic particles). ${ }^{[20]}$ Another possible application of PNJs is atomic trapping and cooling. ${ }^{[21]}$ In this case, extra-long PNJs cancel the influence of the Casimir-Polder potential near the surface of microparticles. Optical forces, acting on a nanoparticle in the vicinity of a subwavelength slit in a metal film were also studied. ${ }^{[22]}$ In refs. [23,24], a so-called "photonic hook" (curved PNJ) is considered and it is theoretically shown that it can be used for particle transport along a curved trajectory around transparent obstacles.

High efficiency of PNJs for nano-object trapping was also confirmed experimentally. ${ }^{[25,26]}$ In these cases, dielectric microspheres attached to a microscope probe produced photonic PNJs to trap and manipulate biological objects, such as blood cells or DNA.

Optical trapping in standing PNJ waves was also reported in refs. $[27,28]$. Two microspheres or microcylinders forming

\author{
A. Kovrov \\ Deprtment of Energy Conversion and Storage \\ Technical University of Denmark \\ DTU Risø Campus \\ Frederiksborgvej 399, 4000 Roskilde, Denmark \\ Dr. A. Novitsky \\ Department of Photonics Engineering \\ Technical University of Denmark \\ Ørsteds Plads 343 \\ DK-2800 Kgs, Lyngby, Denmark \\ Dr. A. Novitsky \\ Department of Theoretical Physics and Astrophysics \\ Belarusian State University \\ Nezavisimosti Avenue 4, 220030 Minsk, Belarus
}

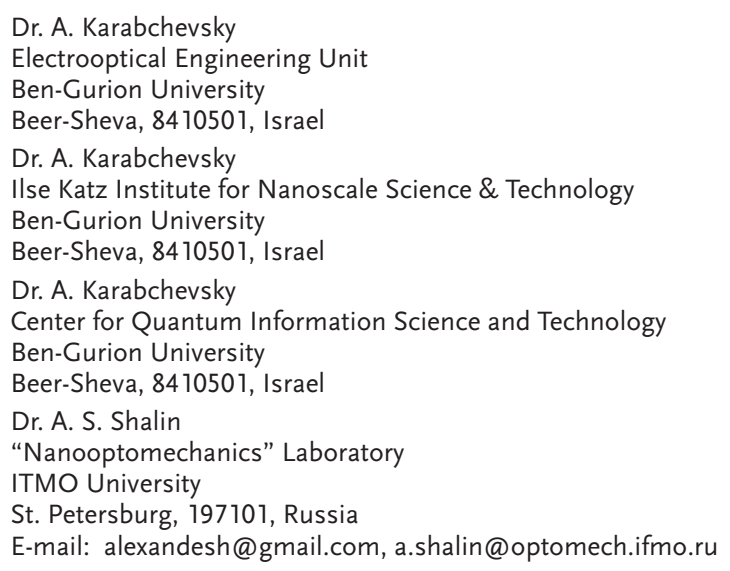


PNJs toward each other produce a stable interference pattern for trapping down to $60 \mathrm{~nm}$ diameter particles in intensity maxima. ${ }^{[27]}$ Alternatively, a reflection geometry can be used to reach the same effect. ${ }^{[28]}$

In this Letter, we theoretically study optical forces in tunable PNJs generated by microspheres and microellipsoids. Although the formation of PNJs in such systems is well known, ${ }^{[29]}$ here we focus on the spatial tuning of the maximum intensity area with the help of wavelength and polarization. We show an application of polarization switching for tunable trapping of dielectric nanoparticles. We show dielectric nanoparticle trapping, and accurate control over their position and longitudinal transport with nanoscale precision. Besides the shift of nanoparticles along the optical axis, we explore their displacement in the perpendicular direction to the PNJ side lobes.

Accurate calculation of the time-averaged optical force acting on a nanoparticle can be carried out using a surface integration of Maxwell's stress tensor. If the particle's size is much less than the wavelength, the optical force is reduced to ${ }^{[3]}$

$$
\langle F\rangle=\frac{\alpha^{\prime}}{4} \nabla E_{0}^{2}+\frac{\alpha^{\prime \prime}}{2} E_{0}^{2} \nabla \varphi
$$

where angle brackets $\langle F\rangle$ denote the time averaging over the period of field oscillations, $\alpha^{\prime}$ and $\alpha^{\prime \prime}$ are real and imaginary parts of the electric polarizability, while $E_{0}$ and $\varphi$ are the amplitude and the phase of the electric field $\mathbf{E}(\mathbf{r})=\mathbf{E}_{0}(\mathbf{r}) \exp [i \varphi(\mathbf{r})]$, respectively. In a most general case, the second term in Equation (1) (scattering force) should be taken into account. However, when nanoparticles are made up of non-absorbing dielectric material and their size is much smaller than the wavelength, the imaginary part of the polarizability is non-zero because of Draine's radiative-reaction correction only. ${ }^{[30]}$ For example, for a nanoparticle of $20 \mathrm{~nm}$ radius and refractive index $n=2, \alpha^{\prime \prime}$ is two orders of magnitude lower than $\alpha^{\prime}$. So, the second term in Equation (1) (scattering force) is usually much lower than the first one (gradient force) in fields with sufficient gradients of intensity. Hereinafter we will consider non-absorbing dielectric nanoparticles and drop Draine's radiative-reaction correction. Then only the first term in Equation (1) is relevant, and we arrive at ${ }^{[3]}$

$\langle F\rangle=\varepsilon_{0} \pi r^{3} \frac{m^{2}-1}{m^{2}+2} \nabla E_{0}^{2}$

where $r$ is the nanoparticle radius, $m=n_{\mathrm{np}} / n_{\mathrm{b}}$ is the relative refractive index, and $n_{\mathrm{np}}$ and $n_{\mathrm{b}}$ are the refractive indices of the nanoparticle and the background material, respectively.

We consider a PNJ generated by a microparticle embedded into a transparent substrate having the same refractive index $n_{\mathrm{b}}$ as a background liquid (Figure 1). The substrate is needed for stabilization of the microparticle's position. Spherical nanoparticles float in an optically homogeneous liquid. Equation (2) can be used to calculate the optical force, acting on the nanosphere, because $r \ll \lambda$.

The microsphere illuminated by a plane wave produces the main lobe of the PNJ and a couple of side lobes in the plane $y=0$ (Figure 2). The electric field amplitude of the PNJ for spherical particles is calculated using Mie theory. The main lobe has a single maximum on the $z$-axis. The side lobe maxima are located

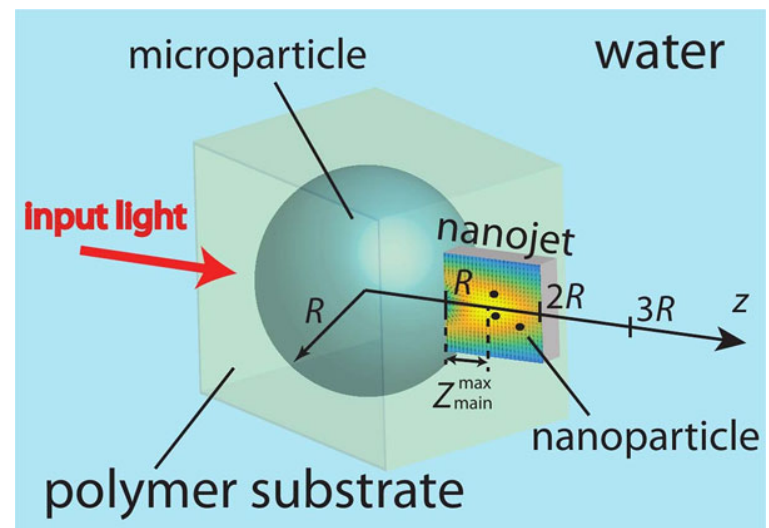

Figure 1. Artistic representation of the PNJ-based light trap. A microparticle is embedded in a polymer substrate, which is in turn embedded in water. Incident light illuminates the microparticle creating a nanojet.

at the surface of the sphere. Side maxima can be very weak as in Figure 2a or, on the contrary, very strong as in Figure 2c. This gives us the opportunity for switching the optical trap between the optical axis and the side lobes. The arrow maps of gradient optical forces in Figure 2 show the positions of the traps for different microsphere's refractive indices $n_{\mathrm{p}}$ : the high-intensity trap moves along the $z$-direction, degrades with appearance of the side traps, and finally all the traps merge into the single trap at the spherical interface. Notice that the direction of the gradient force (Figure 2) is invariant with respect to the size and refractive index of the nanoparticle if these parameters are in the range of the dipole approximation applicability (Equations (1) and (2)) and so the length of the arrows is given in arbitrary units.

Here the field distribution of the PNJ is characterized by the main lobe maximum position $Z_{\text {main }}^{\max }$ (the distance between the microsphere interface and peak intensity at the main lobe as depicted in Figure 1) and the difference between squared peak electric fields for the main $E_{\mathrm{m}}^{2}$ and the side $E_{\mathrm{s}}^{2}$ lobes as

$\Delta=E_{\mathrm{m}, \max }^{2}-E_{\mathrm{s}, \max }^{2}$

According to Figure 2e, fone can move a dielectric nanoparticle along the $z$-axis by changing $\lambda$, while the range of manipulation is determined by $n_{\mathrm{p}}$. The range of values for $n_{\mathrm{p}}$ is determined by frequently used microparticle materials such as titania $\left(\mathrm{TiO}_{2}\right)$ $\left(n_{\mathrm{p}}=2.4\right)$, polystyrene $\left(n_{p}=1.59\right)$, barium titanate $\left(n_{\mathrm{p}}=1.9\right)$. Increasing $n_{\mathrm{p}}$ for a fixed wavelength, the rays are more strongly bent by the microsphere and, hence, the hot spot at the $z$-axis draws closer to the sphere's interface (see Figure 2e). Now the main and side lobes interact with each other (see Figure $2 \mathrm{~b}$ ) and the energy transfers to and fro among them, creating a complicated system of resonances for higher refractive indices $n_{\mathrm{p}}$. Larger wavelengths for a fixed $n_{\mathrm{p}}$ decrease the size parameter $n_{\mathrm{b}} 2 \pi R / \lambda$, which is equivalent to a decrease in the sphere radius $R$. As per Figure 2f, the focus position along the $z$-axis downscales for such effectively smaller microspheres.

Although wavelength switching provides wide opportunities, polarization switching is much easier from an experimental point of view. We consider a spheroidal microparticle that generates the PNJ with an $x$-oriented axis and circular cross section in the $(y, z)$ plane as shown in Figure 3c. When an incident 

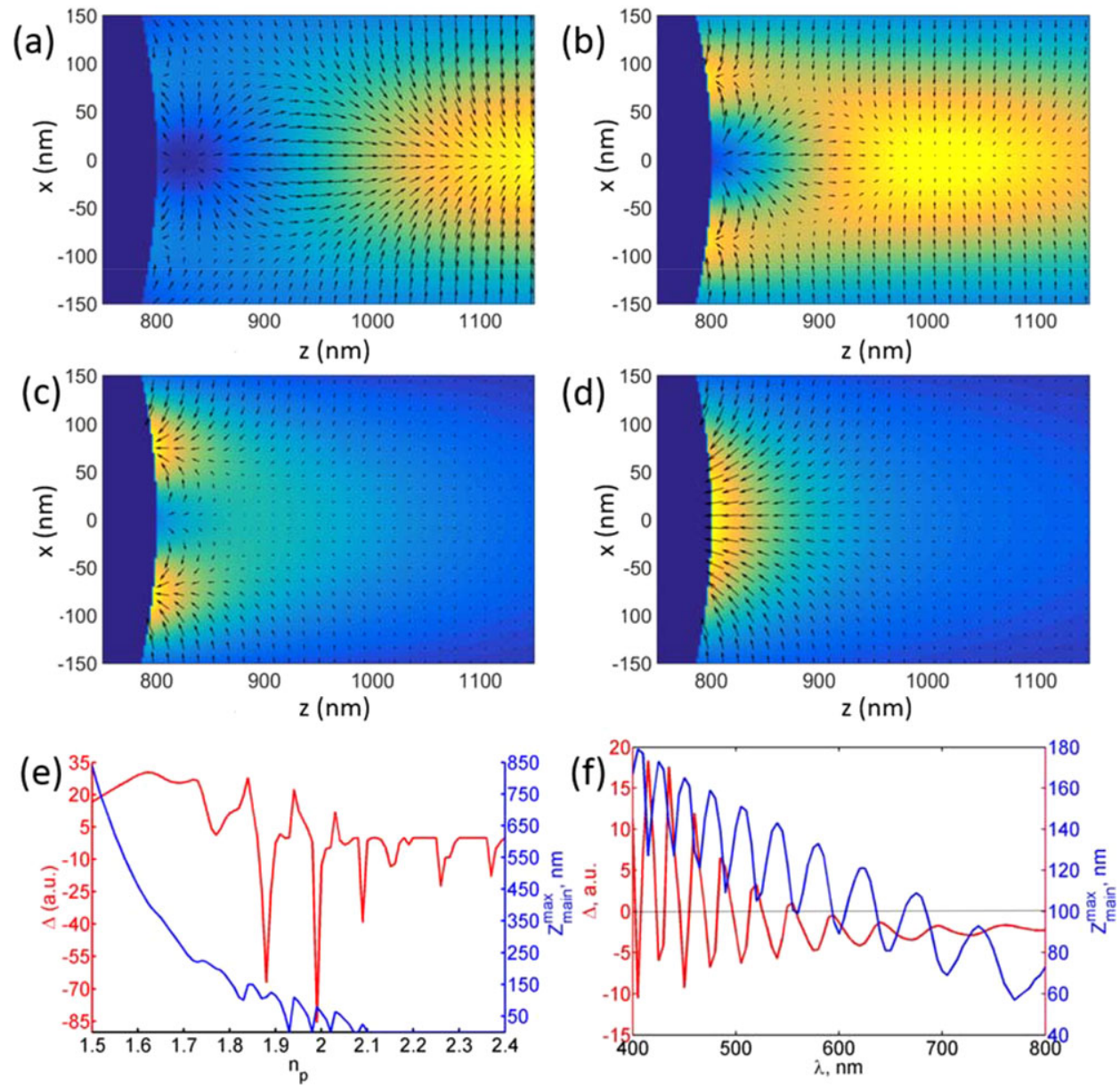

Figure 2. Electric field intensity (color map) and optical force (arrows) acting on dielectric nanoparticles in the PNJ field for: a) $n_{p}=1.62$, b) $n_{p}=1.76$, c) $n_{p}=1.99$, and d) $n_{p}=2.20(\lambda=400 \mathrm{~nm})$. The field intensity inside of the microparticle is not shown. e) $\Delta$ and $Z_{\text {main }}^{\text {max }}$ as a function of $n_{p}(\lambda=$ $400 \mathrm{~nm})$. f) $\Delta$ and $Z_{\operatorname{main}}^{\max }$ as function of $\lambda\left(n_{p}=1.8\right), R=800 \mathrm{~nm}$. Calculated using Mie theory (LightScatPro).

plane wave propagates along the main $z$-axis, the two linear polarizations ( $x$ - and $\gamma$-polarizations) result in apparently different PNJs, and hence, different optical forces. For some aspect ratio $\sigma=\frac{R_{x}}{R_{y}}=\frac{R_{x}}{R_{z}}$ the $x$-and $\gamma$-polarized light can be concentrated in side and main lobes, respectively (see Figure $3 \mathrm{~b}$ ), where $R_{x}$ and $R_{Y}=R_{z}$ are the spheroid's semiaxes. The electric field amplitude of the PNJ for ellipsoidal particles is calculated numerically using Comsol Multiphysics.

For switching purposes we need a noticeable contrast between the $x$ - and $y$-polarizations. From Figure $3 \mathrm{~b}$ we extract the optimal aspect ratio $\sigma=1.15$ corresponding to the maximum contrast. The PNJ intensity maps in the case of a prolate ellipsoid at $\sigma=1.15$ are depicted in Figure 3c,d for the $\gamma$ - and $x$-polarizations, respectively. The nanoparticles trapped by the main lobe in the $\gamma$-polarized light are released and attracted by the side lobe traps, when polarization is switched. If one more switching is made, the particles will return to the main lobe.

The trapping stability with respect to Brownian motion can be characterized with a stability number ${ }^{[31]}$

$S=\frac{W}{k_{B} T}$ where $W$ is the work, $k_{\mathrm{B}}$ is the Boltzmann constant, and $T$ is the temperature (we use room temperature $T=300 \mathrm{~K}$ ). We employ two stability numbers. The ordinary stability number $S_{1}$ characterizes the ability of the trap to avoid escape of nanoparticles due to Brownian motion and exploits the work $W_{1}$ required for transfer of the nanoparticles from the main lobe optical trap to an infinitely remote distance. The stability number $S_{2}$ estimates how another trap may facilitate or obstruct the escape, and utilizes the work $W_{2}$ needed to displace a nanoparticle from the main to the secondary maximum. According to ref. [32], the minimum stability number required for successful trapping equals 10 . Using this assumption we can see from Figure $3 e$ that the nanoparticle's dimensions should be greater than approximately $50 \mathrm{~nm}$ for the optimized ellipsoid and $60 \mathrm{~nm}$ for the optimized sphere. In Figure 3f, we observe that an $x$-polarized PNJ forbids escaping to the side lobes, while a $\gamma$-polarized PNJ weakens the trap and the jump to the side lobe becomes more likely. It is worth mentioning that in the absence of a microsphere a Gaussian beam with corresponding field amplitude cannot provide any trapping for particles of such size.

To summarize, we studied the optical forces exerted on nanoparticles in the PNJ field and showed its tunability with 

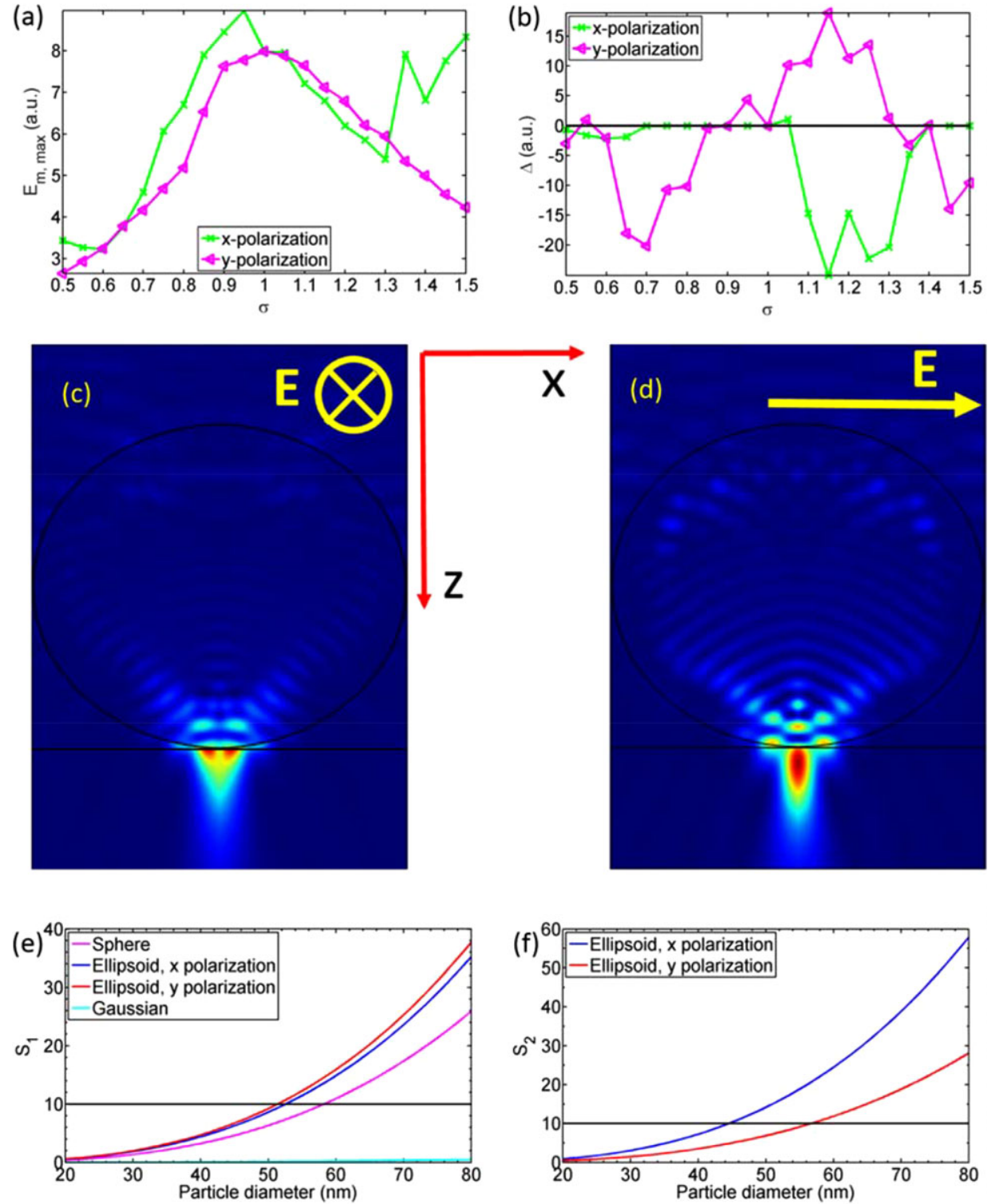

Figure 3. a) The maximum electric field $E_{m, \max }$ in the PNJ main lobe, and b) the difference $\Delta$ as a function of aspect ratio $\sigma$ for the spheroidal microparticle $\left(R_{y}=R_{z}=800 \mathrm{~nm}\right.$ and $\left.n_{p}=2\right)$. c,d) Distribution of the light intensity as a result of interaction between the $x$-polarized (c) and $y$-polarized (d) incident plane wave $(\lambda=400 \mathrm{~nm})$ and microellipsoid $\left(R_{y}=R_{z}=800 \mathrm{~nm}, R_{x}=920 \mathrm{~nm}\right.$, and $\left.n_{\mathrm{p}}=2.15\right)$. e,f) Stability numbers $S_{1}(\mathrm{e})$ and $S_{2}(\mathrm{f})$ versus nanoparticle's diameter $\left(n_{\mathrm{np}}=1.6\right)$ in the PNJ created by the Gaussian beam (peak field value $E_{0}=3 \times 10^{6} \mathrm{~V} \mathrm{~m}^{-1}$ ) and plane-wave field $\left(E_{0}^{\mathrm{pw}}=3 \times 10^{6} \mathrm{~V} \mathrm{~m}^{-1}\right.$ and $\left.\lambda=400 \mathrm{~nm}\right)$ scattered by the optimized microsphere $\left(R=800 \mathrm{~nm}\right.$ and $\left.n_{\mathrm{p}}=1.8\right)$ and optimized microellipsoid $\left(R_{y}=R_{z}=800 \mathrm{~nm}, R_{X}=920 \mathrm{~nm}\right.$, and $\left.n_{\mathrm{p}}=2.15\right)$. Calculations were carried out using COMSOL Multiphysics.

respect to refractive index, wavelength, and polarization. We revealed optical traps in the main and side lobes of the PNJ and demonstrated its ability to move nanoparticles within the traps by adjusting the wavelength of the incoming light. For the first time we have elaborated the conditions for lateral motion (perpendicular to the PNJ propagation direction) of a nanoparticle due to the interplay between main and side lobes of a PNJ. We found that pronounced polarization switching of the position of the main optical trap in a PNJ is generated by ellipsoidal microparticles, and we have determined stability conditions for the optical traps with respect to Brownian motion. We showed that the trapping stiffness provided by a strongly confined PNJ is much higher compared to conventional optical tweezers because of the higher field localization. The results obtained can be applied for designing compact, tunable, and polarization-sensitive PNJ-based optical tweezers supporting precise control over the position and mechanical motion of nanoparticles.

\section{Acknowledgements}

The authors gratefully acknowledge the financial support provided by Russian Fund for Basic Research (Project Nos. 18-02-00414, 18-5200005); Ministry of Education and Science of the Russian Federation (Grant No. 3.4982.2017/6.7); Belarusian Republican Foundation for 
Fundamental Research (Grant No. F18R-021); Multidisciplinary Program Health-Engineering Sciences grant by the Ben-Gurion University of the Negev 2018. The force calculations were partially supported by Russian Science Foundation (Grant No. 18-72-10127).

\section{Conflict of Interest}

The authors declare no conflict of interest.

\section{Keywords}

near-field optics, optical tweezers, optomechanics, photonic nanojet

Received: April 17, 2018

Revised: July 26, 2018

Published online: August 20, 2018

[1] M. L. Juan, M. Righini, R. Quidant, Nat. Photonics 2011, 5, 349.

[2] J. Zhang, K. F. MacDonald, N. I. Zheludev, Phys. Rev. B 2012, 85, 205123.

[3] L. Novotny, B. Hecht, Principles of Nano-Optics, Cambridge University Press, New York 2006, p. 419.

[4] N. Berkovitch, P. Ginzburg, M. Orenstein, J. Phys. Condens. Matter 2012, 24, 073202.

[5] T. Shoji, Y. Tsuboi, J. Phys. Chem. Lett. 2014, 5, 2957.

[6] A. S. Shalin, S. V. Sukhov, Plasmonics 2013, 8, 625.

[7] A. S. Shalin, P. Ginzburg, P. A. Belov, Y. S. Kivshar, A. V. Zayats, Laser Photonics Rev. 2014, 8, 131.

[8] M. I. Petrov, S. V. Sukhov, A. A. Bogdanov, A. S. Shalin, A. Dogariu, Laser Photonics Rev. 2016, 10, 116.
[9] A. Ivinskaya, M. I. Petrov, A. A. Bogdanov, I. Shishkin, P. Ginzburg, A. S. Shalin, Light Sci. Appl. 2017, 6, e 16258.

[10] A. S. Shalin, S. V. Sukhov, A. A. Bogdanov, P. A. Belov, P. Ginzburg. Phys. Rev. A 2015, 91, 063830.

[11] A. A. Bogdanov, A. S. Shalin, P. Ginzburg, Sci. Rep. 2015, 5, 15846.

[12] Z. G. Chen, A. Taflove, V. Backman, Opt. Express 2004, 12, 1214.

[13] A. Heifetz, S.-C. Kong, A. V. Sahakian, A. Taflove, V. Backman, J. Comput. Theor. Nanosci. 2009, 6, 1979.

[14] T. Jalali, D. Erni, J. Mod. Opt. 2014, 61, 1069.

[15] Y. E. Geints, A. A. Zemlyanov, E. K. Panina, J. Opt. Soc. Am. B 2015, 32, 1570.

[16] C.-Y. Liu, Appl. Opt. 2015, 54, 8694.

[17] S. S. Stafeev, V. V. Kotlyar, A. Feldman, Appl. Opt. 2014, 53, 5322.

[18] D. McCloskey, J. J. Wang, J. F. Donegan, Opt. Express 2012, 20, 128.

[19] B. S. Luk'yanchuk, R. Paniagua-Domínguez, I. Minin, O. Minin, Z. Wang, Opt. Mater. Express 2017, 7, 1820.

[20] X. Cui, D. Erni, C. Hafner, Opt. Express 2008, 16, 13560.

[21] V. Yannopapas, Opt. Commun. 2012, 285, 2952.

[22] F. Valdivia-Valero, M. Nieto-Vesperinas, Opt. Commun. 2013, 294, 351.

[23] L. Yue, O. V. Minin, Z. Wang, J. N. Monks, A. S. Shalin, I. V. Minin, Opt. Lett. 2018, 43, 771.

[24] A. S. Ang, A. Karabchevsky, I. V. Minin, O. V. Minin, S. V. Sukhov, A. S. Shalin, Sci. Rep. 2018, 8, 2029.

[25] Y.-C. Li, H.-B. Xin, H.-X. Lei, L.-L. Liu, Y.-Z. Li, Y. Zhang, B.-J. Li, Light Sci. Appl. 2016, 5, e16176.

[26] Y. Li, H. Xin, X. Liu, Y. Zhang, H. Lei, B. Li, ACS Nano 2016, 10, 5800.

[27] H. Wang, X. Wu, D. Shen, Opt. Lett. 2016, 41, 1336.

[28] I. V. Minin, O. V. Minin, V. Pacheco-Peña, M. Beruete, Quantum Electron. 2016, 46, 555.

[29] M.-S. Kim, T. Scharf, S. Mühlig, C. Rockstuhl, H. P. Herzig, Opt. Express 2011, 19, 10206.

[30] B. T. Draine, Astrophys. J. 1988, 333, 848.

[31] A. H. J. Yang, D. Erickson, Nanotechnology 2008, 19, 045704.

[32] H. Wang, X. Wu, D. Shen, Opt. Lett. 2016, 41, 1652. 